\title{
Relevance of the Mo-precursor State in H-ZSM-5 for the Methane Dehydroaromatization
}

Received 00th January 20xx, Accepted 00th January 20xx

DOI: $10.1039 / x 0 x \times 00000 x$
Ina Vollmer ${ }^{\mathrm{a}}$, Guanna Li ${ }^{\mathrm{a}}$, Irina Yarulina ${ }^{\mathrm{a}, \mathrm{d}}$, Nikolay Kosinov ${ }^{\mathrm{b}}$, Emiel J. Hensen ${ }^{\mathrm{b}}$, Klaartje Houben ${ }^{\mathrm{c}}$, Deni Mancec, Marc Baldusc ${ }^{c}$, Jorge Gascon ${ }^{a, d}$, Freek Kapteijn ${ }^{a}$

While the local geometry of Mo in Mo/HZSM-5 has been characterized before, we present a systematic way to manipulate the configuration of Mo and link it to its catalytic properties. The location and geometry of cationic Mo-complexes, the precursor of the active metal site for Methane Dehydroaromatization, is altered by directing the way they anchor to the framework of the zeolite. The handle used to direct the anchoring of Mo is the location of Al in the zeolite framework. According to DFT calculations, the local geometry of Mo should change, while UV-Vis and Pyridine FTIR indicated differences in the dispersion of Mo. Both aspects, however, did not influence the catalytic behavior of Mo/HZSM-5, indicating that as long as enough isolated Mo species are present inside the pores of the zeolite, catalytic behavior is unaffected. This paves the way to better understand how the Mo oxo precursor transforms to the active phase at reaction conditions

\section{Introduction}

Interest and demand for a direct way to convert methane to valuable products is growing with the increasing availability of $\mathrm{CH}_{4}$ sources and the inevitable depletion of oil. Such a process would enable flexible, on the spot, valorisation of small-scale sources of methane, for example as by-product of oil fields which is currently flared or reinjected. ${ }^{1}$ To date, methane is utilized via syngas chemistry, which is only economic at a large scale. ${ }^{2}$ Oxidative methane coupling has attracted vast interest, but has inferior carbon efficiency when compared to nonoxidative methane conversion, because of the high selectivity towards $\mathrm{CO}_{2}$ and $\mathrm{CO}$. Since the pioneering work of Bragin and later Wang et al., 3, 4 the direct aromatization of methane under reducing conditions has attracted a great deal of interest in the scientific community. In this process methane is converted to aromatics, mostly benzene as well as naphthalene, toluene and xylenes with simultaneous production of hydrogen. Ethylene and ethane are also produced in small quantities. The direct aromatization of $\mathrm{CH}_{4}$ only occurs at harsh conditions, because of significant thermodynamic limitations. With a $\Delta G_{r}^{0}=+433 \mathrm{~kJ} / \mathbf{m o l}$ and $\Delta \boldsymbol{H}_{\boldsymbol{r}}^{\mathbf{0}}=+\mathbf{5 3 1} \mathbf{~ k J} / \mathbf{m o l}$ it is only above 650 ㅇ C that conversion of $\mathrm{CH}_{4}$ becomes significant. ${ }^{5}$ The process has not been commercialized up to date. This is, to a large extent, due to the rapid deactivation of the Mo/HZSM- 5 catalyst used (the best

a. Catalysis Engineering, Chemical Engineering Department, Delft University of Technology, Van der Maasweg 9, $2629 \mathrm{HZ}$ Delft, The Netherlands.

b. Schuit Institute of Catalysis, Laboratory of Inorganic Materials Chemistry, Eindhoven University of Technology, P.O. Box 513, 5600 MB Eindhoven, The Netherlands.

c. NMR Spectroscopy, Bijvoet Center for Biomolecular Research, Department of Chemistry, Utrecht University, Pandualaan 8, 3584 CH Utrecht, The Netherlands d. King Abdullah University of Science and Technology, KAUST Catalysis Center Advanced Catalytic Materials, Thuwal 23955, Saudi Arabia

E-mail: jorge.gascon@kaust.edu.sa

Electronic Supplementary Information (ESI) available: See DOI: $10.1039 / x 0 x \times 00000 x$ performing system so far) and to several important issues related with its regeneration. ${ }^{6-8}$

Literature commonly agrees that methane is activated on Mo, which is present in a unique and highly dispersed form inside the channels of HZSM-5. However, Mo was shown to reduce in the beginning of the reaction suggesting that the reduced Mospecies is the active phase for methane dehydroaromatization. ${ }^{9-14}$ Thus far, it is not entirely understood how the Mo oxide geometry of the as-synthesized catalyst transforms to the Mo carbide at reaction conditions and how the initial oxide structure influences the final carbide phase. Podkolzin et. al. have used DFT calculations to find reasonable structures for the Mo carbide phase. ${ }^{15}$ Certainty about the presence of these or other (oxy-)carbide structures at reaction conditions can only be reached by spectroscopic or other experimental observations, which are difficult to perform. Understanding this unique configuration is however crucial to guide Mo/HZSM-5 synthesis and to design new materials with similar catalytic behaviour.

Regarding the influence of the Mo precursor on activity, the most important prerequisite is its presence inside the pores as isolated mono-16 or bi-atomic ${ }^{17}$ entities. ${ }^{18}$ Because it is difficult to characterize the reduced Mo-species at reaction conditions, scientists often rely on characterizing the as-synthesized catalyst. The local geometry around Mo in Mo/HZSM-5 has been characterized by UV Raman using DFT calculations to match structures to the observed vibrations. ${ }^{16}$ The dispersion of Mo has been investigated by UV-Raman ${ }^{19}$, UV-Vis 16, 20, 21, $X^{X}{ }^{2} S^{19,} 22$ and XPS 7,23 . Both geometry and dispersion of the Mo-phase have been speculated to be important for catalysis. ${ }^{16,18,19,21,24,25}$

To shed more light on the relation between these well dispersed Mo oxo units and catalytic performance, a promising approach is to manipulate their structure. To get a direct handle on the volatile Mo oxo species migrating into the pores during the synthesis is near to impossible. Thus, in this paper we chose to manipulate their siting instead. Since Mo is immobilized on the Brønsted acid sites (BASs) of the zeolite, 
which are made up of trivalent $\mathrm{Al}$ in the framework, Mo can be directed by synthesizing zeolites with those BAS in varied locations. With this, we present a systematic way to manipulate the local geometry of Mo and link it to its catalytic properties. ${ }^{16}$ We aim to further understand which property of the precursor state: location, geometry or isolation is most important for the final performance of the Mo precursor.

\section{Experimental Details}

\section{Catalyst Preparation}

The synthesis procedure for the samples with low, medium and high concentration of Al pairs denoted HZ-L, HZ-M and HZ$\mathrm{H}$ respectively was adapted from Dědecěk et. al.. ${ }^{26}$ Solutions for the Al source, the Si source and the structure-directing agent (SDA) were prepared. The Al source solution was either $\mathrm{Al}\left(\mathrm{NO}_{3}\right)_{3} \cdot 9 \mathrm{H}_{2} \mathrm{O}$ in $\mathrm{Dl}$-water (softened to $0,1^{\circ} \mathrm{D}$, deionized via reverse-osmosis via a resistance of $>1 \mathrm{Mohm} / \mathrm{cm}$, disinfected through an UV filter) keeping $\mathrm{H}_{2} \mathrm{O}: \mathrm{Al}\left(\mathrm{NO}_{3}\right)_{3} \cdot 9 \mathrm{H}_{2} \mathrm{O}$ at 126 (solution A) or $\mathrm{AlCl}_{3} \cdot 6 \mathrm{H}_{2} \mathrm{O}$ in DI-water with $\mathrm{H}_{2} \mathrm{O}: \mathrm{AlCl}_{3} \cdot 6 \mathrm{H}_{2} \mathrm{O}=$ 128 (solution $\mathrm{B}$ ). The Si source solution contained tetraethyl orthosilicate (TEOS) with ethanol keeping ethanol:TEOS $=1$ and the SDA containing solution was tetrapropylammonium hydroxide (TPAOH, $1 \mathrm{M}$ in $\mathrm{H}_{2} \mathrm{O}$ ) and DI-water keeping $\mathrm{H}_{2} \mathrm{O}: \mathrm{TPAOH}=101$. In a typical synthesis, two solutions are mixed for $90 \mathrm{~min}$ after which the third solution is added to mix for another $90 \mathrm{~min}$ at ambient conditions. For HZ-L, Al-source solution $A$ is mixed with the $\mathrm{Si}$ source solution first and then the SDA solution is added. For HZ-M, the Si source solution is mixed with $\mathrm{Al}$ source solution $\mathrm{B}$ and then the SDA is added. $\mathrm{HZ}$ $\mathrm{H}$ was synthesized in the same manner as $\mathrm{HZ}-\mathrm{M}$ only adding the SDA prior to the Si source. The Si/TPAOH ratio was kept at 2.65 and $\mathrm{Si} / \mathrm{Al}$ at 25 for all syntheses. Subsequently, hydrothermal synthesis was performed at $170{ }^{\circ} \mathrm{C}$ in $45 \mathrm{ml}$ Teflon liners in steel autoclaves with rotation for 10 days in the case of $\mathrm{HZ}-\mathrm{L}$ and $\mathrm{HZ}-\mathrm{H}$. To obtain the same crystal size for all samples, a shorter duration for hydrothermal synthesis of 7 days was applied for HZ-M. The synthesized crystals were recovered by centrifugation and washing with DI-water 3 times. The so obtained crystals were left for drying overnight at $80{ }^{\circ} \mathrm{C}$ and then calcined at $550{ }^{\circ} \mathrm{C}$ for $7 \mathrm{~h}$, heating at a rate of $2^{\circ} \mathrm{C} / \mathrm{min}$.

$\mathrm{Na}$ ion exchange was achieved with $200 \mathrm{ml} 1 \mathrm{M} \mathrm{NaNO}_{3}$ solution per $1 \mathrm{~g}$ zeolite. The zeolite was exchanged for $7 \mathrm{~h}$ at $80{ }^{\circ} \mathrm{C}$ and afterwards recovered by centrifugation. Almost full $\mathrm{Na}$ ion exchange was achieved as confirmed by ICP (Table S1). The samples were exchanged with $\mathrm{Co}\left(\mathrm{NO}_{3}\right)_{2}$ following $\mathrm{Na}$ exchange. The zeolite was left stirring in $200 \mathrm{ml} 0.05 \mathrm{M}$ solution per gram for $7 \mathrm{~h}$ at $80{ }^{\circ} \mathrm{C}$ and subsequently washed with DI-water and centrifuged 3 times. Co ion-exchange was repeated 3 times. Samples exchanged with Co are denoted by CoHZ- $x$, where $x$ denotes the parent zeolites with a low (L), medium (M) or high ( $\mathrm{H}$ ) concentration of $A l_{\text {pairs. }}$.

Mo was introduced to the acid form of the zeolite through Incipient Wetness Impregnation (IWI) dissolving appropriate amounts of ammonium heptamolybdate (AHM) in a volume of water needed to fill the pores of the zeolite powder $(210 \mu \mathrm{l} / \mathrm{g})$. The samples are dried overnight at $80^{\circ} \mathrm{C}$ and calcined at $550{ }^{\circ} \mathrm{C}$ for $7 \mathrm{~h}$ using a heating rate of $2^{\circ} \mathrm{C} / \mathrm{min}$. Samples are thereafter denoted as $\boldsymbol{y M o H z - x}$, where $y$ denotes the wt.\% of Mo introduced. Catalysts were prepared with 2, 5 and 7.5 wt.\% of Mo.

\section{Catalyst characterization}

Low secondary Electron Imaging (LEI) was used on a JEOL JSM7500F Scanning Electron Microscope field emission scanning electron microscope (SEM) to produce high quality images of the catalysts. An acceleration voltage of $5 \mathrm{kV}$ was used during image acquisition.

Chemical composition of the samples in terms of $\mathrm{Mo}, \mathrm{Co}, \mathrm{Si}$ and $\mathrm{Al}$ content was measured by digestion of approximately 50 mg sample in $4.5 \mathrm{ml} 30 \% \mathrm{HCl}+1.5 \mathrm{ml} 65 \% \mathrm{HNO}_{3}+0.2 \mathrm{ml} 40 \%$ $\mathrm{HF}$ using a microwave. The digestion time in the microwave was $60 \mathrm{~min}$ at max. power. After digestion, the samples were diluted to $50 \mathrm{ml}$ with $\mathrm{MQ}$ and analysed with ICP-OES on a PerkinElmer Optima 5300 (torch:Si+saffire injector). For $\mathrm{Na}$ a PerkinElmer AAS Modell AAnalyst 200 was used.

$X$-ray powder diffraction (XRD) data was collected on a Bruker D8 Advance diffractometer, operating in Bragg-Brentano geometry using Co $K \alpha$ radiation $(\lambda=0.179 \mathrm{~nm})$ and a Lynxeye position sensitive detector to collect data in the range of $2 \vartheta$ from $5^{\circ}$ to $50^{\circ}$ with a scan-speed of $0.2^{\circ} \mathrm{s}^{-1}$ and a sample rotation rate of $30 \mathrm{rpm}$.

$\mathbf{N}_{\mathbf{2}}$ adsorption was performed on a TriStar II 3020 Version 3.02 (Micromeritics) at liquid nitrogen temperature, $T=77 \mathrm{~K}$ for determination of the BET area. The $t$-plot method was used to determine the micropore volume. Before adsorption, the catalyst was outgassed under a flow of $\mathrm{N}_{2}$ at $350^{\circ} \mathrm{C}$ for $16 \mathrm{~h}$.

Pyridine Transmission FTIR spectroscopy was performed on a Nicolet 6700 spectrometer with a MCT/B detector. The sample was first activated in vacuum at $400^{\circ} \mathrm{C}$ for $16 \mathrm{~h}$ to remove adsorbed species. After activation, the pyridine gas was fed to the pellets until saturated and further evacuated at $160{ }^{\circ} \mathrm{C}$ for $2 \mathrm{~h}$. Spectra were recorded in $1000-4000 \mathrm{~cm}^{-1}$ range at $4 \mathrm{~cm}^{-1}$ resolution and co-addition of 128 scans. The spectra shown represent the subtraction result of the spectra collected before adsorption of pyridine from the one taken afterwards. All spectra were normalized by the framework absorbance at $1873 \mathrm{~cm}^{-1}$

The UV-Vis diffuse reflectance spectra (UV-Vis-DRS) were collected on a Perkin-Elmer Lambda 900 spectrophotometer equipped with an integrating sphere ("Labsphere") in the 200$800 \mathrm{~nm}$ range. $\mathrm{BaSO}_{4}$ was used as a white standard for $\mathrm{CoHZ}-x$ and the bare zeolite for $y \mathrm{MoHz}-x$.

Before measurement, the samples were degassed at $400{ }^{\circ} \mathrm{C}$ under $\mathrm{N}_{2}$ flow for $12 \mathrm{~h}$ and then transferred to the sample holders in the glovebox. The absorption intensity is expressed by the Schuster-Kubelka-Munk equation $F\left(R_{\infty}\right)=$ $\left(1-R_{\infty}\right)^{2} / 2 R_{\infty}$

Quantification of $\boldsymbol{A} \mathbf{I}_{\text {pairs }}$ and determination of their location is reported by Dědecěk et. al. $A I_{F}$ exists either as single $A l$ or in pairs. ${ }^{26} \mathrm{Co}$ (II) ions selectively exchange with the Al in pairs at 
the applied exchange conditions. ${ }^{27}$ Therefore, the following formula can be applied to calculate the number of $A I_{F}$ in pairs.

$$
A I_{\text {pairs }}=2 * \mathrm{Co}_{\text {total }}
$$

In addition, because Co replaces $\mathrm{Na}$, the number of Al-pairs can also be calculated by the following formula

$$
\begin{aligned}
& A I_{\text {single }}=N a_{\text {total }} \\
& A I_{F}=A I_{\text {single }}+A l_{\text {pairs }}
\end{aligned}
$$

Where $\mathrm{Co}_{\text {total }}$ and $\mathrm{Na}_{\text {total }}$ represent the total amounts of exchanged $\mathrm{Co}^{2+}$ and $\mathrm{Na}^{+}$cations present in the samples as determined by ICP. Co(II) ions can be either located in the straight or sinusoidal channels of the zeolite or at the intersection of the two. The distribution of $A l_{\text {pairs }}$ among those sites is determined by deconvoluting the absorption bands of Co(II) in UV-Vis with Gaussians.

\section{Catalytic Testing}

Catalytic testing was performed in a quartz tube with an inner diameter of $6 \mathrm{~mm}$, using $500 \mathrm{mg}$ catalyst pelletized to pellets of 212 to $425 \mu \mathrm{m}$. A weight hourly space velocity (WHSV) of $1.21 \mathrm{~h}^{-1}$ was applied in all tests. Product analysis was achieved on an Interscience Trace GC with a TCD and two FIDs. A mixture $5 \% \mathrm{~N}_{2}$ in $\mathrm{CH}_{4}$ was fed to the reactor. The reactor was brought to reaction temperature under the same flow with a heating rate of $10{ }^{\circ} \mathrm{C} / \mathrm{min}$. Although trace amount of many products were detected in the calculations the major products were considered (hydrogen, ethylene, ethane, propylene, benzene, toluene, xylenes and naphthalene).

Product yields were calculated according to (4).

$$
Y_{\text {product }}[\mathrm{mol} \%]=\frac{F_{C_{i} H_{j}}}{F_{C_{4}, i n}} * i * 100 \%
$$

\section{DFT calculations}

Periodic DFT calculations were performed using the Vienna Ab initio Simulation Package (VASP). ${ }^{28,} 29$ The exchangecorrelation energy was described by the generalized gradient approximation PBE functional. ${ }^{30} \mathrm{~A}$ plane-wave basis set with a cut-off of $500 \mathrm{eV}$ in combination with the projected augmented wave (PAW) method was used. ${ }^{31}, 32$ Brillouin zonesampling was restricted to the $\Gamma$ point. The orthogonal MFI unit cell with lattice parameters of $a=20.241 \AA, b=20.015 \AA$, and $c=13.439 \AA$ as optimized by DFT with an all-silica MFI periodic model was used for all calculations. The optimized unit cell parameters agree well with experimental data for calcined ZSM-5. ${ }^{33}$ To compensate for the positive charge of the extra-framework cationic Mo-oxo complexes, one or two framework $\mathrm{Si}^{4+}$ ions in MFI unit cell were substituted by $\mathrm{Al}^{3+}$.

\section{Results}

In the Mo/HZSM-5 system, Mo oxide replaces the proton that balances trivalent $\mathrm{Al}$ in the HZSM-5 framework. The geometry of Mo oxide and its anchoring can be controlled by manipulating the location of $\mathrm{Al}$ in the framework. This $A I_{F}$ siting was directed by the synthesis procedure, namely the source of Al used as well as the order of mixing of structure directing agent (SDA), Al source and silica source (see Table 1). Structural properties from $\mathrm{N}_{2}$ Adsorption are summarized in Table 1, while the morphology is shown in the SEM images in

Figure 1. All zeolites synthesized exhibited the typical diffraction pattern of MFI (Figure S1). Structural properties (Figure S2), morphology and particle size are very similar for all three synthesized HZSM-5 zeolites. In agreement with the similar structural properties, the three samples show virtually identical ${ }^{27} \mathrm{Al}$ ssNMR spectra (Figure S3) where the majority of $\mathrm{Al}$ is in a tetrahedral conformation and about 4-5\% present as extra-framework Al. Only for $\mathrm{HZ}-\mathrm{H}$ the peak corresponding to

\begin{tabular}{|c|c|c|c|c|c|c|}
\hline Sample & Synthesis & Si/Al & $\begin{array}{c}S_{\text {external }} \\
\mathrm{m}^{2} / \mathrm{g}\end{array}$ & $\begin{array}{l}S_{\text {micro }} \\
\mathrm{m}^{2} / \mathrm{g}\end{array}$ & $\begin{array}{l}S_{\text {total }} \\
\mathrm{m}^{2} / \mathrm{g}\end{array}$ & $\begin{array}{l}V_{\text {micro }} \\
\mathrm{cm}^{3} / \mathrm{g}\end{array}$ \\
\hline $\mathrm{HZ}-\mathrm{L}$ & $\mathrm{Al}\left(\mathrm{NO}_{3}\right)_{3} \cdot 9 \mathrm{H}_{2} \mathrm{O}$, TEOS, TPAOH & $24.2 \pm 2.4$ & 31 & 377 & 408 & 0.15 \\
\hline $\mathrm{HZ}-\mathrm{M}$ & TEOS, $\mathrm{AlCl}_{3} \cdot 6 \mathrm{H}_{2} \mathrm{O}, \mathrm{TPAOH}$ & $24.5 \pm 2.4$ & 31 & 381 & 413 & 0.15 \\
\hline $\mathrm{HZ}-\mathrm{H}$ & TPAOH, $\mathrm{AlCl}_{3} \cdot 6 \mathrm{H}_{2} \mathrm{O}$, TEOS & $22.0 \pm 2.2$ & 34 & 378 & 412 & 0.15 \\
\hline
\end{tabular}
tetrahedral Al is shifted by $0.5 \mathrm{ppm}$, which can be explained by the slightly lower Si/Al ratio.

Table 1: Synthesis mixture, Si/Al, and structural properties of the three synthesized zeolites.
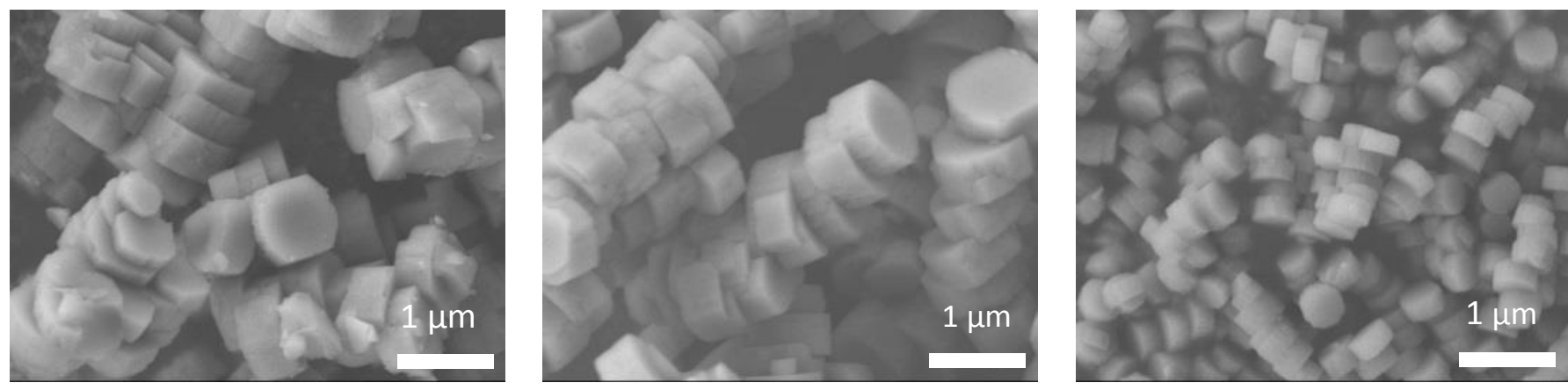

Figure 1: SEM images of HZ-L (left) HZ-M (middle) HZ-H (right). 

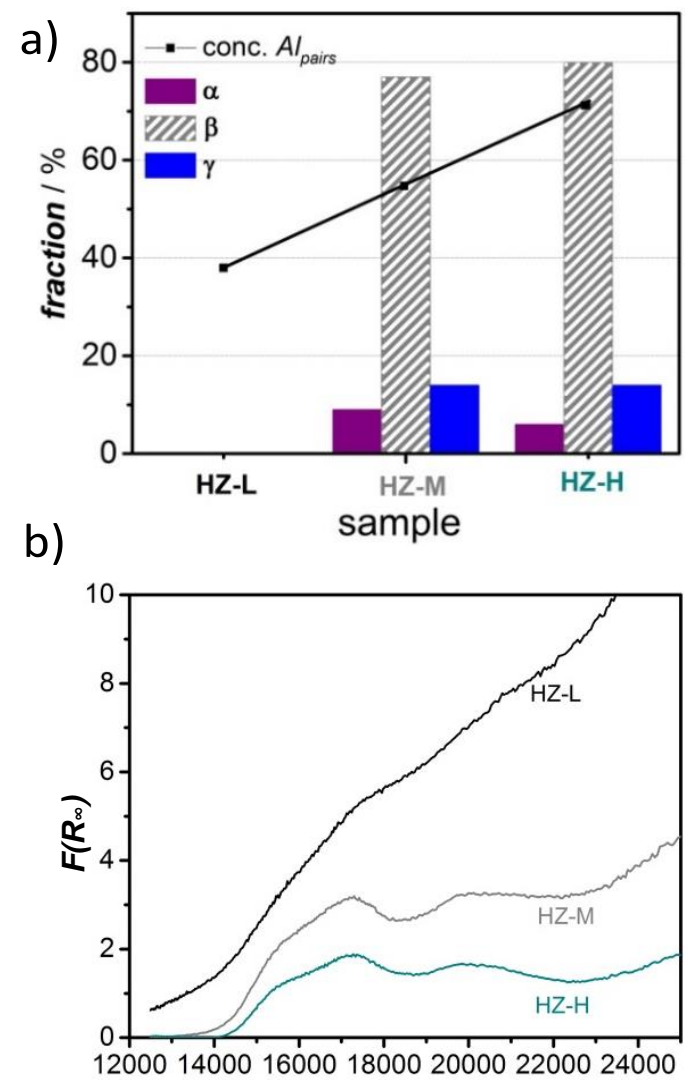

c) $\sigma / \mathrm{cm}^{-1}$

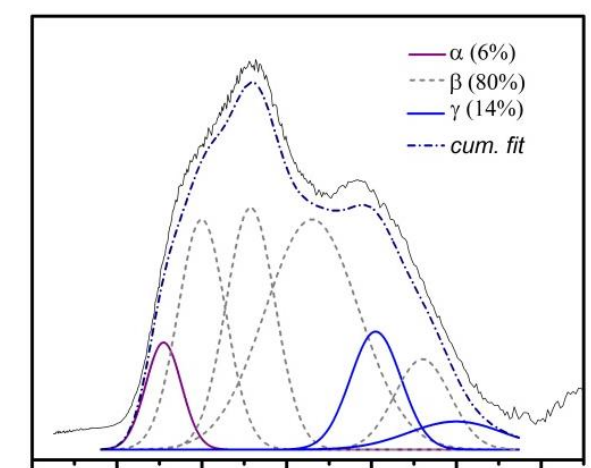

12000140001600018000200002200024000

d)

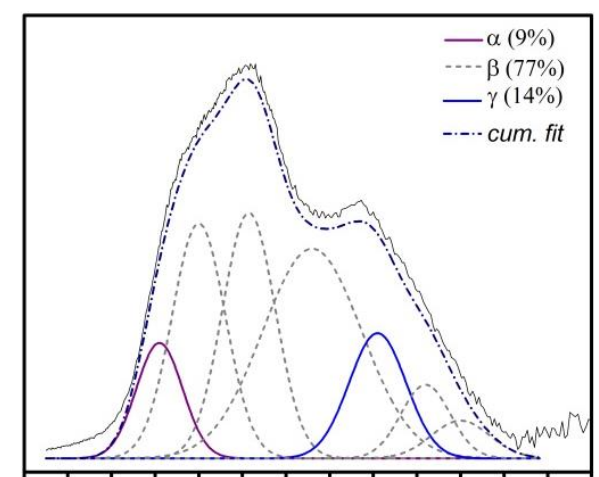

12000140001600018000200002200024000 $\sigma / \mathrm{cm}^{-1}$

Figure 2: Percentage of $\mathrm{Al}$ in $A l_{\text {pairs }}$ calculated using Eq. (2) and the distribution of $A l_{\text {pairs }}$ in $\alpha$-, $\beta$ - and $\gamma$-sites from deconvolution of UV-Vis DRS spectra (a); UV-Vis spectra with deconvolution using Gaussians for $\mathrm{HZ}-\mathrm{H}$ (c) and $\mathrm{HZ}-\mathrm{M}$ (d).
However, the three HZSM-5 differ greatly in terms of $\mathrm{Al}$ location. The three samples have different fractions of $A l$ in pairs $\left(A l_{\text {pairs }}\right) v s$. $A I_{\text {single. }}$. Where $A l_{\text {pairs }}$ consists of two framework Al $\left(A l_{F}\right)$ separated by two Si-O units ( $\left.\mathrm{Al}-\mathrm{O}-(\mathrm{Si}-\mathrm{O})_{2}-\mathrm{Al}\right)$ and $A l_{\text {single }}$ is an $\mathrm{Al}$ separated from another $\mathrm{Al}$ by more than two $\mathrm{Si}-\mathrm{O}$ units. Figure 2a shows that HZ-L has the lowest percentage of $A l_{\text {pairs }}\left(38 \%\right.$ of $A I_{F}$ ) and $\mathbf{H Z}-\mathbf{H}$ the highest $\left(72 \%\right.$ of $A I_{F}$ ), while $\mathbf{H Z}$ $\mathbf{M}$ lies in-between. This is determined by Eq. (2) according to the method developed by Dědecěk and co-workers to identify the location of $A I_{F}$ using cobalt (Co) exchange. Figure $\mathbf{2 b}$ shows the UV-Vis absorption of all three samples, where the intensity of the bands characteristic for Co cations increase from HZ-L to $\mathbf{H Z}-\mathbf{H}$. We further utilized these bands to locate the $A I_{\text {pairs }}$ in $\alpha$, $B$ and $\gamma$ sites, positioned in the straight channel, at the intersection of straight and sinusoidal channels and in the sinusoidal channels, respectively. UV-Vis spectra and Gaussian deconvolution of those bands are shown in Figure $\mathbf{2 c - d}$. Figure 2a summarized the distribution of $A l_{\text {pairs }}$ among the $\alpha, \beta$ and $\gamma$ sites for HZ-M and HZ-H obtained from Gaussian deconvolution, where $\alpha$-sites are identified with the band at $15100 \mathrm{~cm}^{-1}, 6$-sites with the bands at 16000, 17150, 18600 and $21200 \mathrm{~cm}^{-1}$, while Co in $\gamma$-sites gives rise to bands 20100 and $22000 \mathrm{~cm}^{-1}$. The bands in $\mathbf{H Z}-\mathbf{L}$ were not intense enough to achieve meaningful results from Gaussian deconvolution. Deconvolution for the other catalysts reveals a similar distribution of $A l_{\text {pairs }}$ among the three different sites, with most $A l_{\text {pairs }}$ located in the $B$-sites at the intersection of the straight and sinusoidal channels of MFI. Only about $20 \%$ of $A I_{\text {pairs }}$ are located in the straight ( $\alpha$-site) and sinusoidal channels ( $\gamma$-site) of the MFI structure.

Mo structures optimized by DFT calculations (Figure 3) show different geometries depending on whether they are anchored on $A l_{\text {pairs }}$ or $A l_{\text {single }}$. Energies relative to the $\gamma$-site calculated for Mo oxide structures anchored to both $A l_{\text {pairs }}$ or $A l_{\text {single }}$ are low for all three sites considered here, namely $\alpha, B$ and $\gamma$. Because of these small energy differences, differences in location of $A I_{F}$ can direct Mo to be present in varying dispersion (Figure 4-5) and geometry. To determine the size of Mo species or rather the number of Mo-O-Mo bonds formed, the edge energy of Mo, obtained from UV-Vis, was compared with known references as shown by Wachs et al. ${ }^{34}$

Figure 4 shows that for all samples $M o$ is present as monomeric species at low (2 wt.\%) Mo loading, corresponding to $\mathrm{Mo} / \mathrm{Al}=0.35$. At higher loadings however, the zeolites show very different dispersion. While a significant amount of $M o$ is still present as monomeric species in $\mathbf{H Z -} \mathbf{L}$, in $\mathbf{H Z - M}$ and $\mathbf{H Z - H}$ Mo already starts forming Mo clusters at $\mathrm{Mo} / \mathrm{Al}=0.9$. The more $A l_{\text {pairs }}$ are present in the sample, the more clustering occurs, because one Mo will bind to two $A I_{F}$ and therefore more $A I_{F}$ is needed to incorporate the same amount of Mo. This is further confirmed by Pyridine FTIR quantification of the Lewis acid sites (LAS) absorbance at $1455 \mathrm{~cm}^{-1}$ and the Brønsted acid sites (BAS) band at $1546 \mathrm{~cm}^{-1}$ shown in Figure 5 (see Figure S4-5 for full spectra). ${ }^{35}$ While the concentration of both LAS and BAS (Figure 5) as well as the acid strength (Figure S6) of the pristine zeolites is similar, upon incorporating $2 \mathrm{wt} . \%$ 
Mo especially BAS decreases to a higher extent for $\mathbf{H Z}-\mathbf{H}$ than for HZ-L. Acidity decreases to $65 \%$ of the initial value for 2 wt.\% Mo loading (Mo/Al $=0.35$, see Table S2 for ratios determined by ICP) for $\mathbf{H Z}-\mathbf{L}$, which means that one $A I_{F}$ is blocked for each Mo incorporated. For HZ-H Brønsted acidity decreases by more than $65 \%$, leading to 1.5 acid sites neutralized per Mo incorporated (see right axis in Figure 5) confirming that Mo binds to $A l_{\text {pairs }}$ in this case. Incorporation of more Mo, does not decrease the acid site concentration any further, suggesting that instead of anchoring to the framework, Mo is increasingly present in clusters. XPS being a surface sensitive technique confirms these findings further (Figure S7). For $5 \mathrm{wt} . \%$ and $7.5 \mathrm{wt} . \%$ loading, a higher concentration of $\mathrm{Mo}$ is seen with this surface sensitive technique than what is determined by the bulk technique ICP (Figure S7, Table S2), which means that a significant amount of Mo is agglomerated at the surface of the zeolite for these high loadings. In contrast, the Mo loadings obtained from ICP and XPS match very well for $2 \mathrm{wt} . \%$ again confirming that Mo is very well dispersed for these catalysts. This was also observed by Liu et al.. ${ }^{22}$

DFT structure optimization (Figure 3) revealed the different geometries for Mo oxide anchoring to $A l_{\text {pairs }}$ vs. $A I_{\text {single. This }}$ difference in geometry arises from the difference of one negative charge present when binding to two vs. one $A I_{F}$ which is compensated by another dangling oxygen.

This systematic approach to altering the geometry and dispersion of Mo finally allows drawing the crucial connection to catalytic performance in MDA. The three zeolites with 2 wt.\% and 5 wt.\% Mo loading were tested at $650{ }^{\circ} \mathrm{C}$. The methane conversion reaches $4.5 \%$ for 2 wt.\% Mo and around
$7 \%$ for 5 wt.\% Mo in the initial period of the reaction, but decreases to about $1 \%$ during the initial 2 hours on stream for both Mo loadings (Figure 6). Benzene is formed with the highest yield, followed by naphthalene. Only negligible amounts of toluene, ethane and ethylene are formed. Within experimental accuracy the catalytic performance (activity, selectivity and deactivation) of all three zeolites is the same. This was also observed when testing the catalysts at 760 ㅇ $\mathrm{C}$ (Figure S12), although deactivation is faster in that case (Figure S13).

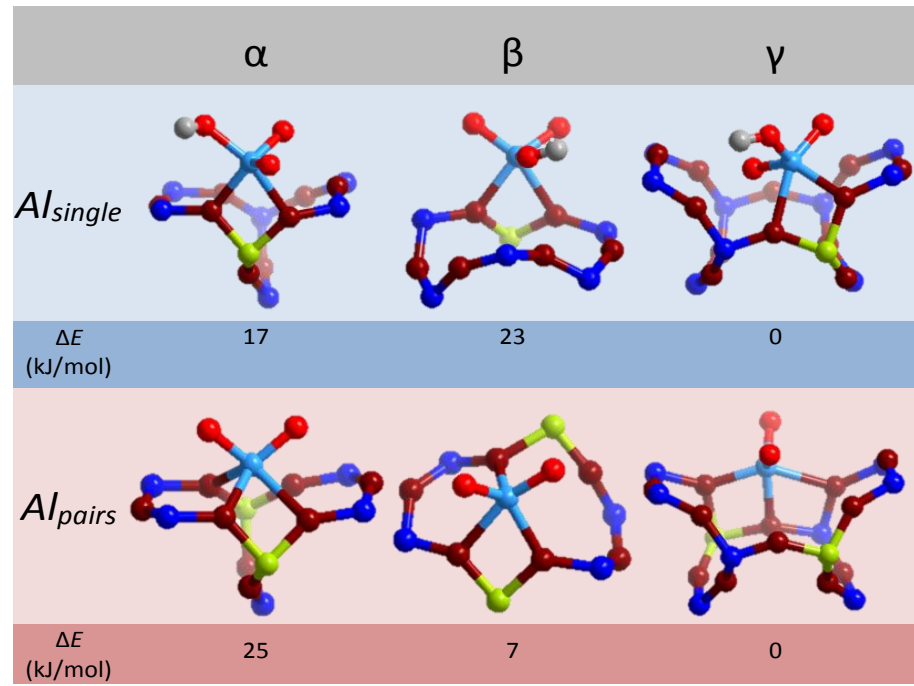

Figure 3: Results of geometry optimization with DFT for Mo oxide anchored either to $A l_{\text {single }}$ (top) or $A l_{\text {pairs }}$ (bottom) in $\alpha, \beta$ and $\gamma$ positions. Energies relative to the $\gamma$-site are given below the images.

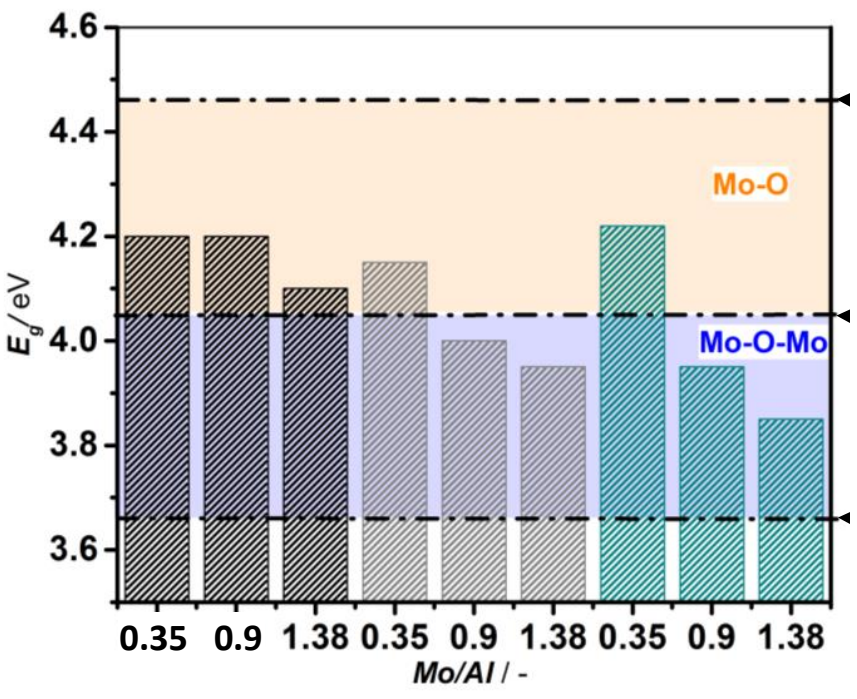

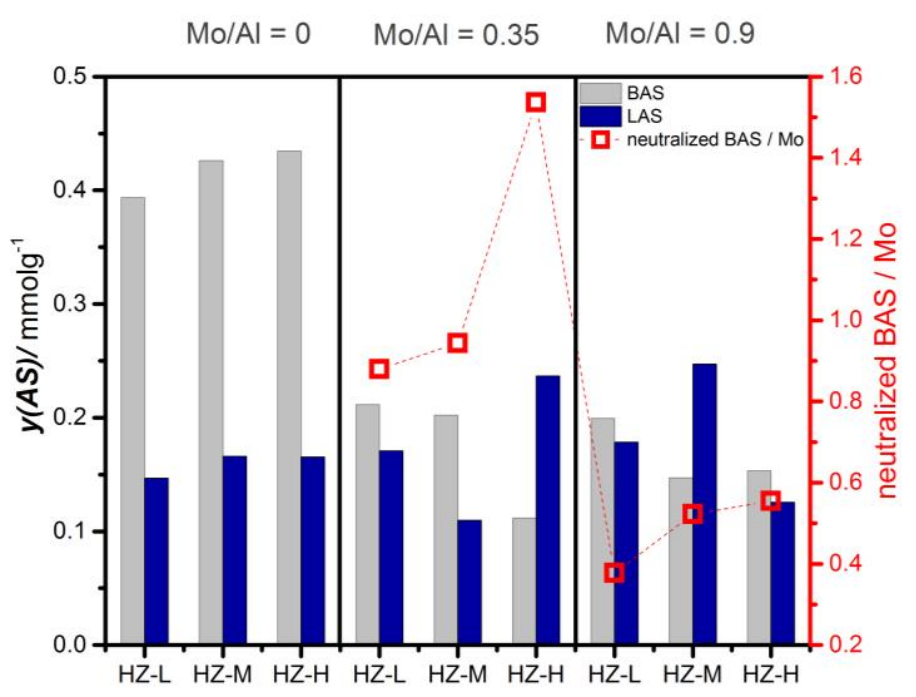

HZ-L HZ-M HZ-H HZ-L HZ-M HZ-H HZ-L HZ-M HZ-H
Figure 4: $E_{g}$ obtained with the Tauc plot method for samples (black: HZ-L, grey: HZ-M, turquoise: $\mathrm{HZ}-\mathrm{H}$ ) with different Mo loadings and references. Full spectra in Figure S8 to 511 .
Figure 5: Concentration of BAS and LAS in HZ-x for different Mo-loading. $\mathrm{Mo} / \mathrm{Al}=0.35$ and 0.9 correspond to $2 \mathrm{wt} . \%$ and $5 \mathrm{wt} . \%$ of Mo respectively. 
a)

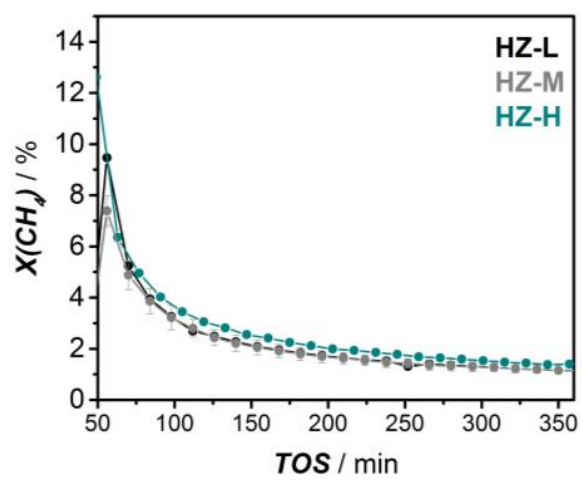

b)

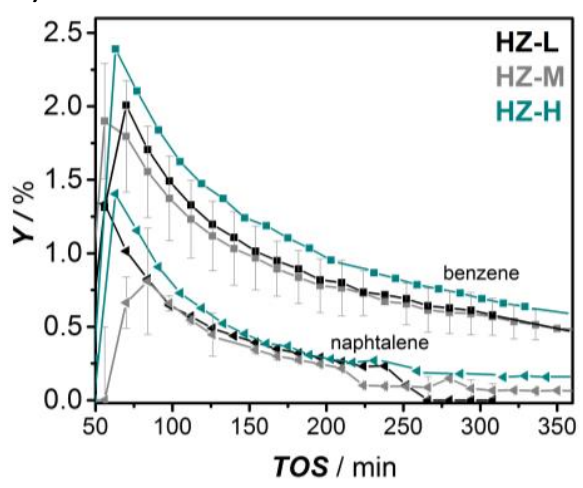

c)

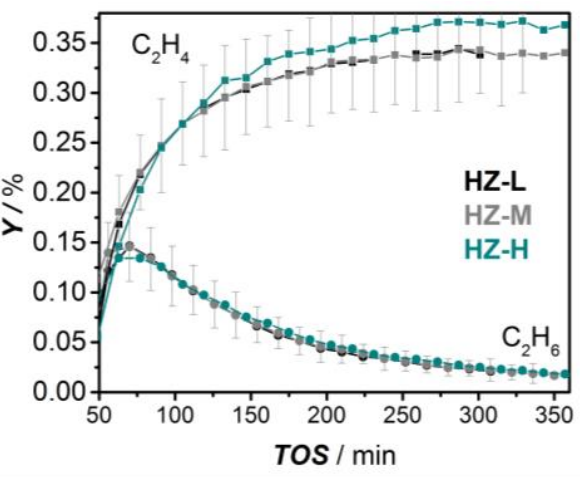

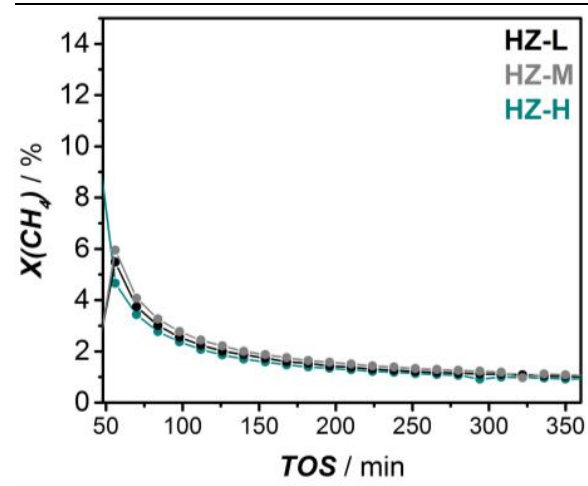
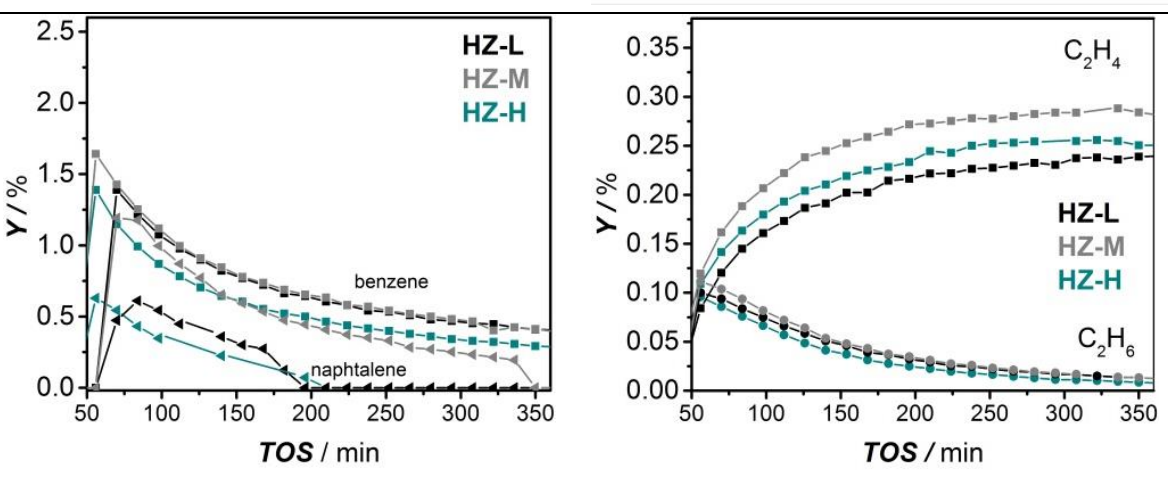

Figure 6: $\mathrm{CH}_{4}$ conversion (a), naphthalene and benzene yield (b), ethylene and ethane yield (c), for $5 \mathrm{MoHZ}-\mathrm{x}($ top $)$ and $2 \mathrm{MoHZ}-\mathrm{x}(\mathrm{bottom})$ at $650{ }^{\circ} \mathrm{C}$ with $\mathrm{WHSV}=1.21$ $\mathrm{h}^{-1}$ and $500 \mathrm{mg}$ catalyst.

This is explained by the fact that the bonds the Mo precursor forms with the framework and its geometry transform significantly at reaction conditions. It was shown that the Mo gets reduced under these conditions which is likely to break at least one bond of the Mo with the framework thereby changing its geometry, as demonstrated for Tungsten. ${ }^{17}$ The catalytic results also imply that both Mo anchoring to $A l_{\text {pairs }}$ as well as $A l_{\text {single }}$ reduce easily enough leading to a similar delay in onset of the reaction. In all catalysts prepared in this work, enough isolated Mo oxo units are inside the pores of the zeolite to lead to similar catalytic behaviour, so that seems to be the more important factor for this particular system.

\section{Conclusions}

These results suggest that the precursor state of the Mo and the aluminium distribution in the three ZSM- 5 samples plays no decisive role in the final performance for methane dehydroaromatization. We attribute this effect to the significant transformation of the local environment and binding of $\mathrm{Mo}$ at reaction conditions. At the reaction temperatures methane converts the Mo oxide into Mo (oxy)carbide, which was shown to be the active phase in this reaction. ${ }^{9-14}$ In this sense, the isolation of the Mo oxo units and their location inside the channels of the zeolite seem to be crucial in the development of an efficient pre-catalysts. The geometry does not play a role, likely because it is transformed upon carburization. With this work, we bridge the gap between the Mo precursor state and catalytic performance. So far work has either focused on extensive characterization of the Mo precursor ${ }^{16,18,21}$ or on measuring the effect of other factors on catalytic performance ${ }^{36-38}$. Our results underscore the necessity to develop appropriate characterization tools for methane dehydroaromatization catalysts that elucidate how the actual catalytic site forms under reaction conditions. ${ }^{21,} 25$

\section{Acknowledgements}

Financial support from the Sabic-NWO CATC1CHEM CHIPP project is gratefully acknowledged. Thanks go to Dr. Christoph Dittrich (SABIC), Dr. Frank Mostert (SABIC) and Dr. T. Alexander Nijhuis (SABIC). G. Li acknowledges financial support from The Netherlands Organization for Scientific Research (NWO) for her personal VENI grant (no. 016.Veni.172.034) and NWO-Surf SARA for providing access to supercomputer resources.

\section{Notes and references}

1. J. H. Lunsford, Catalysis Today, 2000, 63, 165-174.

2. J. Raskó and J. Kiss, Applied Catalysis A: General, 2003, 253, 427-436.

3. L. Wang, L. Tao, M. Xie, G. Xu, J. Huang and Y. Xu, Catal Lett, 1993, 21, 35-41.

4. O. Bragin, T. Vasina, Y. I. Isakov, B. Nefedov, A. Preobrazhenskii, N. Palishkina and K. M. Minachev, Russ Chem Bull, 1982, 31, 847-847. 
5. S. Ma, X. Guo, L. Zhao, S. Scott and X. Bao, Journal of Energy Chemistry, 2013, 22, 1-20.

6. K. Honda, X. Chen and Z.-G. Zhang, Catalysis Communications, 2004, 5, 557-561.

7. N. Kosinov, F. J. Coumans, G. Li, E. Uslamin, B. Mezari, A. S. Wijpkema, E. A. Pidko and E. J. Hensen, Journal of Catalysis, 2017, 346, 125-133.

8. A. Jens, B. Rainer and M. Leslaw, in Small-Scale Gas to Liquid Fuel Synthesis, CRC Press, 2015, DOI: doi:10.1201/b18075-2110.1201/b18075-21, pp. 531-560.

9. H. Jiang, L. Wang, W. Cui and Y. Xu, Catal Lett, 1999, 57, 95-102.

10. I. Lezcano-González, R. Oord, M. Rovezzi, P. Glatzel, S. W. Botchway, B. M. Weckhuysen and A. M. Beale, Angewandte Chemie International Edition, 2016, 55 5215-5219.

11. F. Solymosi, A. Szöke and J. Cserényi, Catal Lett, 1996, 39 , 157-161.

12. D. Wang, J. Lunsford and M. Rosynek, Top Catal, 1996, 3, 289-297.

13. S. Liu, L. Wang, R. Ohnishi and M. Lchikawa, Kinetics and catalysis, 2000, 41, 132-144.

14. W. Ding, S. Li, G. D Meitzner and E. Iglesia, The Journal of Physical Chemistry B, 2001, 105, 506-513.

15. J. Gao, Y. Zheng, G. B. Fitzgerald, J. de Joannis, Y. Tang, I. E. Wachs and S. G. Podkolzin, The Journal of Physical Chemistry C, 2014, 118, 4670-4679.

16. J. Gao, Y. Zheng, J.-M. Jehng, Y. Tang, I. E. Wachs and S. G. Podkolzin, Science, 2015, 348, 686-690.

17. W. Ding, G. D. Meitzner, D. O. Marler and E. Iglesia, The Journal of Physical Chemistry B, 2001, 105, 3928-3936.

18. J.-P. Tessonnier, B. Louis, S. Walspurger, J. Sommer, M.-J. Ledoux and $\mathrm{C}$. Pham-Huu, The Journal of Physical Chemistry B, 2006, 110, 10390-10395.

19. J. P. Thielemann, T. Ressler, A. Walter, G. Tzolova-Müller and C. Hess, Applied Catalysis A: General, 2011, 399, 2834.

20. R. Kumar Rana and B. Viswanathan, Catal Lett, 1998, 52, 25-29.

21. C. Sun, S. Yao, W. Shen and L. Lin, Catal Lett, 2008, 122, 84-90.

22. S. Liu, L. Wang, R. Ohnishi and M. Ichikawa, Journal of Catalysis, 1999, 181, 175-188.

23. B. S. Liu, L. Jiang, H. Sun and C. T. Au, Applied Surface Science, 2007, 253, 5092-5100.

24. J.-Z. Zhang, M. A. Long and R. F. Howe, Catalysis Today, 1998, 44, 293-300.

25. D. Wang, J. H. Lunsford and M. P. Rosynek, Journal of Catalysis, 1997, 169, 347-358.

26. J. Dedecek, V. Balgová, V. Pashkova, P. Klein and B. Wichterlová, Chemistry of Materials, 2012, 24, 3231-3239.

27. J. Dědeček and B. Wichterlová, The Journal of Physical Chemistry B, 1999, 103, 1462-1476.

28. G. Kresse and J. Hafner, Physical Review B, 1993, 47, 558.

29. G. Kresse and J. Furthmüller, Computational Materials Science, 1996, 6, 15-50.

30. J. P. Perdew, K. Burke and M. Ernzerhof, Physical review letters, 1996, 77, 3865.

31. G. Kresse and D. Joubert, Physical Review B, 1999, 59, 1758.

32.
33.

34.

35.

D. $V$

36.

37.

37.

Sur

H. Van Koningsveld, J. Jansen and H. Van Bekkum, Zeolites, 1990, 10, 235-242.

H. Tian, C. A. Roberts and I. E. Wachs, The Journal of Physical Chemistry C, 2010, 114, 14110-14120.

I. Yarulina, S. Bailleul, A. Pustovarenko, J. R. Martinez, K. D. Wispelaere, J. Hajek, B. M. Weckhuysen, K. Houben, M. Baldus, V. Van Speybroeck, F. Kapteijn and J. Gascon, ChemCatChem, 2016, 8, 3057-3063.

J.-P. Tessonnier, B. Louis, S. Rigolet, M. J. Ledoux and C. Pham-Huu, Applied Catalysis A: General, 2008, 336, 79-88. A. Martínez, E. Peris and A. Vidal-Moya, in Studies in Surface Science and Catalysis, eds. P. M. Antoine Gédéon and B. Florence, Elsevier, 2008, vol. Volume 174, Part B, pp. 1075-1080.

38. H. Liu, Y. Li, W. Shen, X. Bao and Y. Xu, Catalysis Today, 2004, 93-95, 65-73. 